\title{
Process evaluation of the implementation of colour-coded wristbands for signalling special patient status on a neurology ward
}

\author{
Bob van de Loo $^{1}$, Nadiyah de la Rambelje ${ }^{2}$, and Jolanda van Haastregt ${ }^{2}$ \\ ${ }^{1}$ Amsterdam UMC - Locatie VUMC \\ ${ }^{2}$ Affiliation not available
}

April 28, 2020

\begin{abstract}
Rationale, aims and objectives: Colour-coded wristbands (CCWs) are widely used to signal special patient status to health care professionals. However, little is known about the feasibility of CCWs. The current study describes a process evaluation of an intervention in which CCWs were used to signal special patient status to health care professionals on the neurology Ward of a university hospital in the Netherlands. In this intervention, CCWs were issued to patients with an increased risk of falling and patients with dysphagia. Methods: Quantitative and qualitative methods were used to evaluate the reach of the intervention, performance according to protocol, compliance to the intervention, staff's opinion on the intervention, and contextual factors that may have affected the implementation of the intervention. Data were gathered by means a self-administered questionnaire among the care professionals on the neurology ward and by means of a semi-structured interview among a subgroup of care professionals who guided the implementation process on the ward. Results: Five care professionals were interviewed and 23 care professionals $(57.5 \%)$ responded to the questionnaire. Most professionals indicated they had received information about the CCWs and their role in conducting the intervention. The intervention was largely performed according to protocol, however some deviations from protocol were reported with respect to how family members were informed of the intervention, how the CCWs were issued, and how often patients were inspected for having the appropriate CCWs. Overall, staff members evaluated the intervention positively and perceived patients to be content with the CCWs. Conclusion: We conclude that the CCWs were largely implemented according to protocol. Our findings highlight some challenges that can arise when implementing CCWs. Recommendations are made to optimize the feasibility of the intervention.
\end{abstract}

\section{Introduction}

Falls and dysphagia (or difficulty swallowing) are common problems among older patients. Falls are the most frequently reported adverse event in acute care settings [1]. Rates of falls range from approximately 2 to 8 falls per 1000 patient-days among hospitalized patients [2]. The proportion of these falls that result in some injury ranges from $30 \%$ to $51 \%$ [3]. Moreover, falls can prolong hospitalization, increase cost of care and induce fear of falling [3]. Dysphagia can cause patients to aspirate food or liquids into their lungs [4]. Approximately $15 \%$ of people older than 65 years of age suffer from dysphagia [4]. Presence of dysphagia at admission is associated with poor outcomes, including poor functional ability, institutionalisation, and increased mortality [5].

Colour-coded wristbands (CCWs) have been implemented in hospitals to prevent falls and mitigate the adverse effects of dysphagia [6]. CCWs are widely used to signal special patient status in order to increase awareness among health care professionals. Over 25 American state hospital associations have provided their hospitals with guidelines on standardized colours for CCWs [7]. Additionally, Sevdalist et al. [8] found that out of 62 hospitals in the British National Health Service they reviewed, at least 42 had implemented CCWs. 
Evidence on the effectiveness of CCWs is limited and contradictory. Forster et al. [9] investigated the use of pink and grey wristbands for indicating the target oxygen saturation of patients. The researchers found the CCWs to be beneficial in improving the safe prescription of oxygen to inpatients. However, two other studies that investigated the effect of CCWs for indicating patients with an increased risk of falling both showed no benefit of the CCWs in reducing the number of falls [10,11]. To our knowledge, there are no studies into the effectivity of CCWs for signalling dysphagia.

In addition to the limited evidence for the effectivity of CCWs, little is known about the feasibility of CCWs in daily practice. Forster et al. [9] report that the CCWs for indicating the target oxygen saturation of patients were positively evaluated by patients and staff members. However, other publications reveal several issues related to the implementation of CCWs, such as: the colour coding may not be understood by care professionals [12], the CCWs may not be visible or seen [12,13], the CCWs may not be issued consistently to patients with a special patient status [14], and the colour coding may be not be standardized between or within hospitals [8]. Poor implementation of CCWs may reduce their effectiveness and may even be hazardous. To illustrate, failure to consistently issue the CCWs according to protocol can cause them to not accurately represent a special patient status [14]. Moreover, a provider's misunderstanding of a colour's meaning can trigger an incorrect response, possibly resulting in an adverse event [13]. Nonetheless, for most of these issues it is unclear how prevalent they are and how they can be best prevented, given that there is little documentation on the implementation of CCWs.

In December 2016, the neurology ward of Maastricht University Medical Center+ (MUMC+), located in Maastricht, the Netherlands, implemented CCWs for patients with an increased risk of falling and for patients with dysphagia. This study aims to evaluate the implementation process of this intervention. Saunders, Evans and Joshi [15] suggest that a process evaluation should focus on the following components of an implementation process: reach, fidelity, dose delivered (i.e., completeness), dose received (i.e., exposure and satisfaction), recruitment, and context. Using these components as a guideline, this process evaluation aims to assess the extent to which the care professionals were informed of the intervention (reach), the extent to which the different components of the intervention were performed according to protocol(fidelity and dose delivered), the extent to which the patients were compliant with the CCWs according to the care professionals(dose received- exposure), the opinion of the care professionals on the CCWs (dose receivedsatisfaction), and the contextual factors affected the implementation of the CCWs (context) .

\section{Methods}

\section{Intervention}

A green wristband to indicate an increased risk of falling and a yellow wristband to indicate the presence of dysphagia were implemented on the neurology ward of the participating hospital in September 2016. The implementation of the CCWs was guided by a project team of ten members, consisting of nurses from the neurology ward and quality managers. In total, 40 health care professionals of the neurology ward were involved in conducting the intervention and were supposed to take preventive measures based on the CCWs. The care professionals included nurses $(\mathrm{n}=31)$, physiotherapists $(\mathrm{n}=5)$, an occupational therapist, speech therapists $(\mathrm{n}=2)$, and a nutritionist. Physicians had a limited role in conducting the intervention as they were not responsible for mobilizing and/or monitoring food intake of patients. Therefore, physicians were not included in this process evaluation. The care professionals were informed of the intervention and their role in conducting the intervention, through a newsletter and regular team meetings.

Of the care professionals involved, nurses have a primary role in conducting the intervention as they are responsible for issuing the CCWs. Nurses have to use a set of standardized questions to determine if patients should be issued a green wristband, and have to administer a water-swallowing test to determine 
if patients should be issued a yellow wristband. In case of changes in the situation of a patient, nurses should re-evaluate whether a CCW needs to be issued. Nurses should verify daily whether all patients with a special patient status are wearing a CCW. When a patient is issued a CCW, nurses need to inform them and their family about the intervention verbally and by handing them a flyer containing further information. In addition to the flyers, further information for patients and family members was also included in tabletop sign holders that were placed in every patient room. The sign holders are three-sided and contain sheets detailing the use and purpose of the CCWs. Instructions related to the issuing of the CCWs were included in the regular training sessions of the nurses. All care professionals involved in the intervention have to determine themselves what preventive measures should be applied to meet the special needs of patients wearing a CCW. The type of preventive measures that should be applied are not prescribed by the project team but depend on the assessment made by the care professionals.

\section{Study design and setting}

This process evaluation employed a multi-method approach, consisting of a case study and a survey. Data collection took place on the neurology ward of the MUMC+ between May and July 2017. The neurology ward can accommodate up to 22 patients. Patients that are cared for in the neurology ward are often cognitively impaired.

\section{Case study}

In the case study, data were gathered by means of semi-structured interviews with members of the project team who guided the implementation process (table 1). A purposive sampling technique was used to recruit the five project members that were most involved in the intervention for participation in the interview. A topic guide was created to guide the interview process. The interviews were conducted face-to-face in a private room in the hospital and were set to last approximately 30 minutes. The interviews were digitally recorded with verbal informed consent of the interviewee and transcribed using an intelligent verbatim style [16]. Anonymity in the reporting of the results was assured to the interviewees. The transcripts were coded using open, axial and selective coding, as described by Boeije [17]. Subsequently, for each interview, a summary was made based on the coding process and sent to the respective interviewee for a member check [17]. The member check helps ensure that the results of the interviews are correctly interpreted by the researcher.

\section{Survey}

For the survey, data were gathered by means of a self-administered questionnaire among the care professionals (table 1). The questionnaires were made available to the nurses in the staff room for a period of two weeks. To increase the response, a reminder was sent to the nurses by email and senior nurses were asked to notify the staff on the questionnaire during staff meetings. The neurology ward's physiotherapists, speech therapists, and nutritionists received the questionnaire in their pigeonhole.

The questionnaire consisted of three sections: one for each wristband and one on the overall implementation of the intervention. The questionnaire contained 38 questions of which 37 were close-ended and one was open-ended. Among the close-ended questions were five questions that measured the frequency at which the intervention's procedures were applied and 16 questions that assessed attitudes towards the intervention, on a five-point Likert scale ranging from 1 (strongly disagree) to 5 (strongly agree) or from 1 (very dissatisfied) to 5 (very satisfied). The open-ended question was used to collect views on how intervention could be improved. Data were processed anonymously.

The answers to the close-ended questions were analysed by means of descriptive techniques. The questions that related to the training sessions, the issuing of the CCWs, and informing patients and their family on the 
intervention, only applied to the nurses. For these questions, only the answers of the nurses were analysed. The responses to the open-ended question were categorized based on matching content.

\section{Research ethics statement}

Medical ethical approval was not necessary, as this study only included care professionals.

\section{Results}

\section{Participants}

All five selected project members agreed to participate in the interview. Among the five project members were four nurses and one quality manager. Two project members were interviewed in the same session at their request. Of the 40 care professionals that work on the neurology ward, $23(57.5 \%)$ responded to the questionnaire. The majority of the respondents were nurses (table 2).

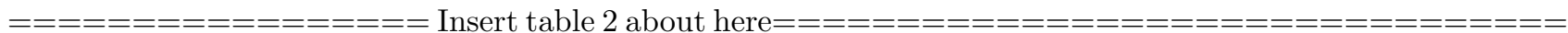

\section{Reach}

Of the five project members who were interviewed, two reported that all targeted staff members were informed of the intervention. Two other project members reported that at least all nurses were informed, but that they were not sure whether all other care professionals were informed. The other project member indicated that staff members who exclusively work during weekends or vacation periods may not have been informed of the CCWs and their use. The results of the questionnaire revealed that $20(87.0 \%)$ and $21(91.3 \%)$ of the care professionals who participated in the survey reported to be informed of the use of respectively the green and yellow wristbands, respectively. Two of the nurses who participated in the survey reported not being informed of either wristbands and one of the speech therapists reported not being informed of the yellow wristband. The survey respondents were asked to rate their satisfaction with the manner in which they were informed of the CCWs. Of the survey respondents who were informed of the CCWs, $13(65.0 \%)$ and $14(66.6 \%)$ reported being (very) satisfied with how they were informed of respectively the green and yellow wristband. The survey respondents were asked to rate the extent to which they agreed with several statements related to the received information. The majority of the respondents reported that their colleagues were aware of the CCWs. Moreover, respondents generally appraised the information received on the CCWs as clear (table 3).

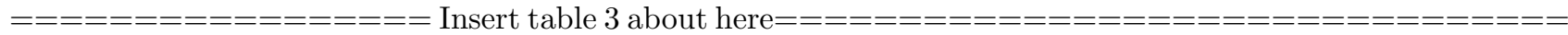

\section{Performance according to protocol}

Results of the questionnaire showed that all 13 nurses who had reported that they had been informed of the CCWs always perform the water-swallowing test when admitting stroke patients. In addition, nine of these nurses reported that in almost all cases patients are issued a CCW after a fall incident and four reported that this happens in most cases. During the interviews, two of the five project members indicated encountering patients at the neurology ward who should have been wearing a CCW but who had not been issued one. According to these project members, nurses sometimes forget to issue the CCWs during hectic periods, such as nightshifts and large transfers of patients. In the questionnaire, the nurses who reported being aware of the CCWs were asked to which extent all patients who need a CCW, as indicated by their electronic health record, actually wear it. Nine of the 13 nurses who were informed of the CCWs reported that almost all patients who need a CCW wear one and four reported that most patients who need it wear one. These nurses were also asked how often they verify whether all patients who need a CCW wear it. Three nurses 
indicated patients are inspected for wearing a CCW every day, four indicated this is done almost every day, five indicated this is done a few times a week, and one indicated not knowing how often this is done.

All of the interviewed project members reported that the sign holders were placed in every patient room, as intended. The questionnaire revealed that five nurses always provide the flyers to the patients with a CCW and/or their family, six nurses provide them to the patients sometimes, and two nurses provide them seldom or never to the patients. The nurses were also asked to what extent they explain the use of the CCWs to patients and their families. The majority of the nurses $(\mathrm{n}=11)$ reported always explaining the use of the CCWs to patients. One nurses reported explaining the use of the CCWs to the patients in some cases and another reported explaining it seldom or never. With regard to explaining the use of the CCWs to family members, seven nurses reported always doing so, four nurses reported doing so most of the times, one nurse reported doing so sometimes and another reported doing so seldom or never.

During the interviews, the four project members who are nurses reported being attentive to the special needs of patients with a CCW. Examples of preventive measures they took for patients wearing green wristbands included: employing motion sensors, putting on non-slip socks, putting the beds in low position, and putting the bed's side rails up. With regard to the yellow wristband, the projected members mentioned a sign that is hung on the beds of patients with a yellow wristband, to remind staff and visitors to not provide food that poses a choking hazard. Project members also reported that the location of patients with a yellow wristband is mapped to aid the nutritionists in their work. In the questionnaire, respondents were asked to rate their level of agreement with several statements related to the performance according to protocol (table 3 ). The respondents generally reported being more careful when mobilizing patients with a green wristband and when offering food and drink to patients with a yellow wristband. However, respondents reported mixed attitudes towards whether they treat patients with a CCW differently.

\section{Compliance}

During the interviews, all five project members indicated that patients sometimes remove their CCWs during a state of deliriousness and that the CCWs are sometimes covered by patients' clothing. In the questionnaire, the nurses were asked how often patients and family members refuse the CCWs. Of the 13 nurses that were informed of the CCWs, three reported the patients sometimes refuse the CCWs and 10 reported the patients refuse them seldom or never. One nurse reported that family members sometimes refuse the CCWs. The other nurses $(\mathrm{n}=12)$ reported that family seldom or never refuse the CCWs. Respondents were asked how often they see patients (un- or intentionally) wear clothes over their CCWs. Of the respondents who were informed of at least one of the CCWs $(n=21)$, eight indicated seeing patients wear clothes over their CCWs often, five reported seeing this regularly, four reported seeing this sometimes, and four reported seeing this seldom or never or not having paid attention to it.

\section{Opinion on the intervention}

In the questionnaire, respondents were asked to express their opinion on the intervention by rating the extent to which they agreed with several statements (table 3). For both CCWs, approximately half of the respondents reported that they think that CCWs help to improve quality of care. Only a minority of the respondents reported that they think that patients find the CCWs bothersome. The respondents reported mixed attitudes towards whether the family of the patients find the CCWs useful. Respondents were asked to rate their satisfaction with the CCWs as an aid. Of the respondents who were informed of the CCWs, $11(55.0 \%)$ and $14(66.7 \%)$ reported being (very) satisfied with respectively the green and yellow wristband as an aid. All of the interviewed project members observed that patients and family members generally are content with the CCWs.

In the interviews and questionnaire, we asked how the intervention could be further improved. During the interviews, three project members suggested implementing a third colour code that represents both the 
green and yellow wristband to help make patients more comfortable. The two other interviewed project members offered no suggestions for improvement. The majority of the surveyed care professionals $(\mathrm{n}=$ $17,74.0 \%$ ) offered no suggestions for improvement. Two survey respondents wrote that they would like the physiotherapists be more involved in issuing the green wristbands. One survey respondent suggested organizing a meeting for all the neurology ward's staff members to establish more clarity on the protocols of the intervention. Another respondent noted that the issue of patients covering their CCWs warrants more attention.

\section{Context}

During the interviews, the project members were asked how contextual factors could have affected the intervention. According to the project members, the implementation of the intervention was postponed because some staff members were concerned that labelling patients with CCWs would violate their privacy. In addition, the project members noted several factors that caused the implementation period to be busier than usual. First, during the implementation period, the hospital was also being accredited by the Netherlands Institute for Accreditation in Healthcare. Second, because of undergoing renovation, the neurology ward had to be temporarily move its activities to a different location in the hospital. Finally, two project members were absent for some time due to private reasons.

\section{Discussion}

This study evaluated the implementation process of CCWs for patients with an increased risk of falling and patients with dysphagia on the neurology ward of a university hospital in the Netherlands. Results from this study indicate most care professionals were informed of the intervention and understood the received information. Overall, the care professionals reported being satisfied with the CCWs and the way they were informed. In addition, this study revealed that care professionals generally considered the special needs of patients with a CCW. However, the care professionals reported some deviations from protocol. First, patients with dysphagia or an increased risk of falling did not always have a corresponding CCW. Nurses seemed to sometimes forget to issue the CCWs during hectic periods and patients sometimes removed the CCWs. Furthermore, it was not verified on a daily basis whether all patients with a special patient status were actually wearing their CCW. Finally, family members of patients were not always informed on the intervention verbally or by being handed the flyer.

Porter et al. [14] assessed the use of CCWs for indicating the presence of allergies in a paediatric emergency department. The researchers found that not all patients with allergies were wearing a CCW. Of the 256 parent-child pairs that were included, there were 28 cases of true allergies according to a guideline-based assessment. Of these 28 cases, only 16 were observed to wear a CCW. Similarly, our findings indicate that in the present intervention not all patients with an increased risk of falling or dysphagia were actually wearing a CCW. Furthermore, our findings on the satisfaction of the CCWs among staff and patients are consistent with the findings of Forster et al. [9]. In the study of Foster, $86 \%$ of the involved staff members reported that the CCWs for indicating patients' target oxygen saturation added at least some benefit and $46 \%$ reported they helped significantly. Of the staff members who were informed of the CCWs in our study, $55 \%$ and $67 \%$ reported being (very) satisfied with respectively the green and yellow wristband as an aid. Moreover, Forster and colleagues reported that the patients that were surveyed in their study $(\mathrm{n}=192)$ generally responded positively. We were unable to directly assess the satisfaction of patients with the CCWs in the present study. Still, in accordance with the results of Forster and colleagues, the observations of the care professionals in the present study suggest that patients generally were content with the green and yellow wristband. Norris et al. [12] describe several risks associated with the implementation of CCWs of which some can be compared with our findings. Our findings substantiate the claim of Norris and colleagues that the CCWs may not be seen by care professionals. Indeed, the care professionals in our study noted that CCWs were regularly covered by patients' clothing. Norris and colleagues also indicate that the colour coding 
of the wristbands may not be understood by the care professionals. Yet, we found that the majority of the care professionals were informed of the intervention. Moreover, the surveyed care professionals who were informed of the intervention generally appraised the received information on the green and yellow wristband as clear. Therefore, our results indicate that the meaning of the colour coding of the wristbands was clear for most care professionals.

This study has several limitations. First, a risk in this type of evaluation studies is that participants may feel inclined to give socially desirable answers. To minimize this risk, we assured anonymity to all study participants in the reporting of the study and conducted the interviews in a private room. Second, our study design was susceptible to recall bias as we relied on self-reported, retrospective data. However, taking direct observations to assess the extent to which the intervention was performed according to protocol was considered too invasive. Third, the scope of this study was limited to evaluating the implementation process of CCWs on only one ward. Hence, it remains unclear to which extent our results are generalizable to other wards and the hospital environment in general. However, it seems likely that the issues related to the implementation of CCWs encountered in the present study are not unique to neurology wards and are relevant to other clinical settings as well. Strong aspects of this study are the use of a multi-method approach and the incorporation of the framework by Saunders et al. [15], which provides a systematic approach for studying implementation processes.

Based on our results, we conclude that although the implementation of CCWs seems feasible in daily practice, several challenges can arise when implementing CCWs. We observed several deviations from protocol which should be addressed when implementing CCWs in daily practice. The following recommendations are made for addressing these challenges when implementing CCWs. First, we recommend taking a rigorous and continuous approach to informing the care professionals of the intervention. Evidently, the colour coding can only provide cues to care professionals that have been informed of the CCWs. As such, guidelines related to the CCWs should be actively communicated to new staff members. Second, we advise to embed the verification process of the CCWs in the daily routines of care professionals. Finally, measures should be taken to ensure that the CCWs are visible for staff members at all times. Wood and Bagaine [13] found that especially the visibility of CCWs is of importance in preventing adverse outcomes. Therefore, care professionals should motivate patients to wear their CCWs in a visible way and notify patients when their CCWs is covered up. More research is needed to establish whether CCWs can help in improving health outcomes. The present study highlights several challenges that arise when implementing CCWs, of which some have been described in previous works. Future research into the effectiveness of CCWs may yield more positive results if these challenges are accounted for.

\section{Acknowledgements}

We would like to thank the staff members of the neurology ward of the Maastricht UMC+ who gave their time to participate in this study.

\section{Conflict of interest}

The authors declare no conflict of interest.

\section{References}

1. Hendrich AL, Bender PS, Nyhuis A. Validation of the Hendrich II Fall Risk Model: a large concurrent case/control study of hospitalized patients. Applied nursing research . 2003;16(1):9-21. doi:10.1053/apnr.2003.YAPNR2

2. Hunderfund ANL, Sweeney CM, Mandrekar JN, Johnson LM, Britton JW. Effect of a Multidisciplinary Fall Risk Assessment on Falls Among Neurology Inpatients. Mayo Clinic Proceedings . 2011;86(1):19-24. doi:10.4065/mcp.2010.0441 
3. Oliver D, Healey F, Haines TP. Preventing Falls and Fall-Related Injuries in Hospitals. Clinics in Geriatric Medicine . 2010;26(4):645-692. doi:10.1016/j.cger.2010.06.005

4. Brady A. Managing the patient with dysphagia. Home healthcare nurse . 2008;26(1):41-46; quiz 47-48. doi:10.1097/01.NHH.0000305554.40220.6d

5. Smithard DG, Smeeton NC, Wolfe CD. Long-term outcome after stroke: does dysphagia matter? Age and ageing . 2007;36(1):90-94. doi:10.1093/ageing/afl149

6. New Jersey Hospital Association. Standardizing Color Codes for Patient Risk Factors. 2007. Available from: http://pdc-media.com/downloads/nj_training_manual.pdf

7. American Hospital Association. Quality Advisory: Implementing Standardized Colors for Patient Alert Wristbands. 2008. Available from: http://www.aha.org/advocacy-issues/tools-resources/advisory/2008/080904quality-adv.pdf

8. Sevdalis N, Norris B, Ranger C, Bothwell S, the Wristband Project T. Designing evidence-based patient safety interventions: the case of the UK's National Health Service hospital wristbands. Journal of evaluation in clinical practice . 2008;15(2):316-322. doi:10.1111/j.1365-2753.2008.01026.x

9. Forster S, Smith S, Daniel P, et al. Optimising prescription and titration of oxygen for adult inpatients using novel silicone wristbands: results of a pilot project at three centres. Clinical medicine (London, England) . 2016;16(4):330-334. doi:10.7861/clinmedicine.16-4-330

10. Mayo NE, Gloutney L, Levy AR. A randomized trial of identification bracelets to prevent falls among patients in a rehabilitation hospital.Archives of physical medicine and rehabilitation . 1994;75(12):1302-1308

11. Vassallo M, Vignaraja R, Sharma JC, et al. The Effect of Changing Practice on Fall Prevention in a Rehabilitative Hospital: The Hospital Injury Prevention Study. Journal of the American Geriatrics Society . 2004;52(3):335-339. doi:10.1111/j.1532-5415.2004.52102.x

12. Norris B, Sevdalis N, Bothwell S, Ranger C, Rose T. The role of patient identification in patient safety. Paper presented at Proceedings of the International Conference on Contemporary Ergonomics; April, 2007; Nottingham.

13. Wood SD, Bagian JP. A Cognitive Analysis of Color-Coded Wristband Use in Health Care. Proceedings of the Human Factors and Ergonomics Society Annual Meeting . 2011;55(1):281-285. doi:10.1177/1071181311551058

14. Porter SC, Manzi SF, Volpe D, Stack AM. Getting the data right: information accuracy in pediatric emergency medicine. Quality 83 safety in health care . 2006;15(4):296-301. doi:10.1136/qshc.2005.017442

15. Saunders RP, Evans MH, Joshi P. Developing a process-evaluation plan for assessing health promotion program implementation: a how-to guide. Health promotion practice . 2005;6(2):134-147. doi:10.1177/1524839904273387

16. Hadley G. Preliminary decisions. In: Grounded Theory in Applied Linguistics Research: A practical guide. 2nd ed. Abingdon: Routledge; 2017:67-85.

17. Boeije H. Analysis in qualitative research. 12th ed. Los Angeles: SAGE; 2010.

\section{Tables}

Table 1 outcome measures of the process evaluation

\section{Component}

Reach

Performance according to protocol (fidelity and dose delivered) 
Compliance (dose received- exposure)

Opinion on the intervention (dose received- satisfaction)

Context

CCW, colour-coded wristband; CS, case study conducted among the members of the project team; ST, survey study condu

Table 2 Characteristics of the survey respondents $(\mathrm{n}=23)$

\section{Characteristic}

Profession Nurse Nurse in training Physiotherapist Nutritionist Speech therapist Time of employment on the neurology war + The intervention had been initiated 6 to 7 months prior to when the questionnaires were distributed.

Table 3 Attitudes of the care professionals towards the questionnaire's statements

\section{Statement}

Reach All my colleagues know what the green wristband indicates All my colleagues know what the yellow wristband indice Performance according to protocol

I am more careful when mobilizing patients with a green wristband

I am more careful when offering food and drink to patients with a yellow wristband

I treat patients with a green wristband differently

I treat patients with a yellow wristband differently

Opinion on the intervention

The green wristbands help me deliver better care

The yellow wristbands help me deliver better care

The patients find the wristbands bothersome

The family of the patients find the wristbands useful

+ Valid percentages are shown to account for missing responses. ++ Respondents who were informed of the CCWs were as 